\title{
Time-accurate solution of stabilized convection-diffusion-reaction equations: II. Accuracy analysis and examples
}

\author{
Antonio Huerta ${ }^{1, *}$ Bernardino Roig ${ }^{2}$ and Jean Donea ${ }^{3}$ \\ ${ }^{1}$ Departament de Matemàtica Aplicada III, E.T.S. de Ingenieros de Caminos, Universitat Politècnica de \\ Catalunya, Jordi Girona 1, E-08034 Barcelona, Spain. \\ 2 Departament de Matemàtica Aplicada, Escola Politècnica Superior de Gandia, Universitat Politècnica de \\ València, Carretera Natzaret-Oliva s/n, E-46730 Grau de Gandia, València, Spain. \\ ${ }^{3}$ LTAS, Thermo-Mécanique, Université de Liège, Campus du Sart-Tilman, Bât. 52,B-4000 Liège, Belgium.
}

\section{SUMMARY}

The paper addresses the development of time accurate methods for solving transient convectiondiffusion-reaction problems using finite elements. The accuracy characteristics of the spatially stabilized implicit multi-stage time stepping schemes developed in a companion paper (Part I of this work) are analyzed and compared here. This is done by means of a Fourier analysis. An important improvement is observed when the order of the method is increased. Moreover, the stabilization techniques proposed (SUPG, GLS, SGS and least-squares) do not degrade the phase accuracy. Finally, some examples are presented to show the applicability of these schemes.

KEY WORDS: convection-diffusion-reaction, time stepping schemes, stabilization, least-squares, finite element method

\section{INTRODUCTION}

In a companion paper [9] implicit multi-stage time stepping schemes of high accuracy are combined with spatial stabilization techniques. A general presentation of the time marching schemes can be found in $[7,8]$. The stabilization techniques proposed are well-known, see for instance $[2,14,3]$. In truly transient convection-diffusion-reaction problems, which are of interest here, and by contrast with the steady state case, the truncation error in the discretization of the linear one-dimensional equation cannot be expressed in the form of a diffusion operator. In transient problems, the overall truncation error depends upon the particular time-stepping method used in combination with finite elements $[12,4,6,5]$ and it generally involves both even and odd spatial derivatives of the unknown, thus simultaneously affecting the dissipative and the dispersive properties of the numerical schemes. Here a Fourier

\footnotetext{
*Correspondence to: Professor Antonio Huerta, E-mail: antonio.huerta@upc.es

Contract/grant sponsor: Ministerio de Ciencia y Tecnología; contract/grant number: REN2001-0925-C03
} 
(von Neumann) analysis is performed in order to study the accuracy of the proposed schemes, see $[1,11,16]$. In particular, the influence of the stabilization schemes on the phase and damping responses is studied. The proposed time stepping schemes are based on diagonal Padé approximations [8], which are A-stable. They are combined with spatial stabilization techniques and numerical stability is guaranteed. This is not the case for the corresponding implicit Runge-Kutta methods of the Lobatto IIIA family. In the final section three examples are presented to validate the proposed methods.

\section{STABILIZED IMPLICIT MULTI-STAGE TIME STEPPING SCHEMES}

\subsection{The partial differential equation}

We are concerned with a time accurate finite element solution of the following convectiondiffusion-reaction equation

$$
u_{t}+\mathcal{L}(u)=s, \quad \text { with } \mathcal{L}:=\boldsymbol{a} \cdot \nabla-\nu \nabla^{2}+\sigma .
$$

The coefficients in $\mathcal{L}$ are assumed constant for the accuracy analysis.

\subsection{The numerical schemes}

As proposed in [9] the consistently stabilized weak form that must be solved is
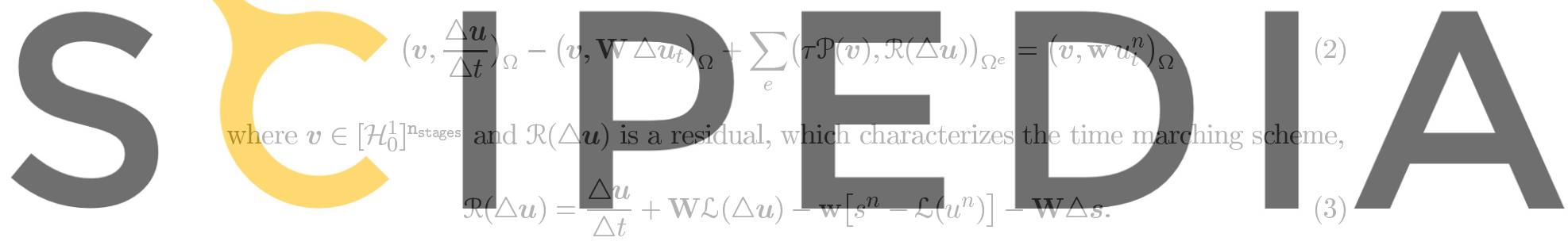

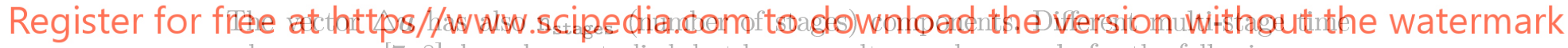
schemes, see [7,8], have been studied, but here, results are shown only for the following:

Second order Padé approximation: $R_{11}$ (Crank-Nicolson)

$$
\begin{aligned}
\triangle \boldsymbol{u} & =u^{n+1}-u^{n}, & \triangle \boldsymbol{s} & =s^{n+1}-s^{n}, \\
\mathbf{W} & =\frac{1}{2}, & \mathbf{w} & =1 .
\end{aligned}
$$

Note that in this case $\mathrm{n}_{\text {stages }}=1$ and, consequently, the vectors and matrix are scalars.

Fourth order Padé approximation: $R_{22}$

$$
\begin{aligned}
\triangle \boldsymbol{u} & =\left\{\begin{array}{c}
u^{n+1 / 2}-u^{n} \\
u^{n+1}-u^{n+1 / 2}
\end{array}\right\}, & \triangle \boldsymbol{s} & =\left\{\begin{array}{c}
s^{n+1 / 2}-s^{n} \\
s^{n+1}-s^{n+1 / 2}
\end{array}\right\}, \\
\mathbf{W} & =\frac{1}{24}\left[\begin{array}{cc}
7 & -1 \\
13 & 5
\end{array}\right], & \mathbf{w} & =\frac{1}{2}\left\{\begin{array}{l}
1 \\
1
\end{array}\right\} .
\end{aligned}
$$


Note the similarity between the above two-stage scheme and the implicit Lobatto IIIA Runge-Kutta method of fourth-order accuracy. While the present scheme is based on nonoverlapping step increments, the corresponding Runge-Kutta method employs total increments such as

$$
\triangle \boldsymbol{u}^{*}=\left\{\begin{array}{c}
u^{n+1 / 2}-u^{n} \\
u^{n+1}-u^{n}
\end{array}\right\}
$$

This minor difference (a linear transformation relates both schemes) does not affect linear problems with a Galerkin formulation. However, as shown in the next section, it has major consequences as regards numerical stability when implemented in connection with stabilized spatial formulations.

\section{ACCURACY ANALYSIS}

The classical von Neumann analysis, see [1, 11, 16], assumes periodic boundary conditions and studies a homogeneous linear differential equation with constant coefficients, i.e. Eq. (1) with $s=0$. The analytical solution for one Fourier mode in $\mathrm{n}_{\mathrm{sd}}$ spatial dimensions reads

$$
u(\boldsymbol{x}, t)=\prod_{j=1, \ldots, \mathrm{n}_{\text {sd }}} e^{-\left(\nu k_{j}^{2}+\sigma\right) t} e^{i k_{j}\left(x_{j}-a_{j} t\right)},
$$
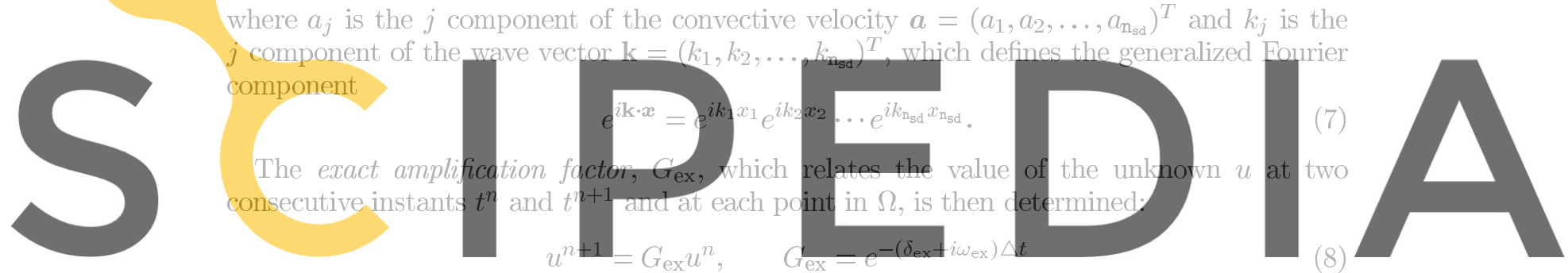

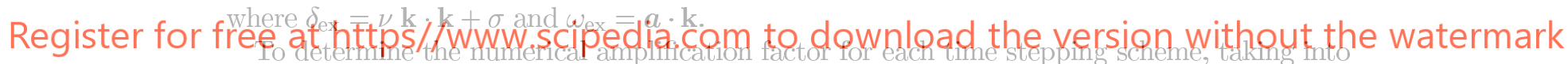
account the spatial discretization, it is necessary to determine the influence of each spatial operator on a Fourier mode. Thus discrete operators associated to the continuous spatiau operators in (2), must be determined. In Table I these relations are shown for uniform linear 1D elements of size $h$ and using the well known difference operators, see [16] for details,

○ Second order centered first difference: $\delta u(x, t)=u(x+h, t)-u(x-h, t)$

○ Second order centered second difference: $\delta^{2} u(x, t)=u(x-h, t)-2 u(x, t)+u(x+h, t)$

To determine the influence of the numerical scheme on a Fourier component, see (7), one introduces the dimensionless wave vector ("number" in the 1D case), $\boldsymbol{\xi}=h \mathbf{k}$. Moreover, using $\triangle t$ and $h$ the parameters in the differential equation, (1), are scaled. The Courant number is $c=\|\boldsymbol{c}\|$ with $\boldsymbol{c}=\boldsymbol{a} \triangle t / h$, the diffusion number is $d=(\nu \triangle t) / h^{2}$, and the dimensionless reaction is $r=\sigma \triangle t$. The exact amplification factor, (8), is rewritten as

$$
G_{\mathrm{ex}}(\boldsymbol{\xi}, \boldsymbol{c}, d, r)=e^{-\left(\delta_{\mathrm{ex}}+i \omega_{\mathrm{ex}}\right) \triangle t}=e^{-(d \boldsymbol{\xi} \cdot \boldsymbol{\xi}+r+i \boldsymbol{c} \cdot \boldsymbol{\xi})} .
$$




\begin{tabular}{|c|c|c|c|}
\hline spatial operator & discrete & mode transf. & expression \\
\hline$\left(N_{i}, N_{j}\right) / h^{2}$ & $1+\frac{1}{6} \delta^{2}$ & $\mathcal{M}(\boldsymbol{\xi})$ & $1+(\cos \xi-1) / 3$ \\
\hline$\triangle t\left(N_{i}, \boldsymbol{a} \cdot \nabla N_{j}\right) / h^{2}$ & $\frac{1}{2} c \delta$ & $\mathcal{A}(\boldsymbol{\xi}, \boldsymbol{c})$ & $i c \sin \xi$ \\
\hline$-\triangle t \nu\left(\nabla N_{i}, \nabla N_{j}\right) / h^{2}$ & $d \delta^{2}$ & $\mathcal{K}(\boldsymbol{\xi}, d)$ & $2 d(\cos \xi-1)$ \\
\hline$\triangle t\left(\boldsymbol{a} \cdot \nabla N_{i}, N_{j}\right) / h^{2}$ & $-\frac{1}{2} c \delta$ & $-\mathcal{A}(\boldsymbol{\xi}, \boldsymbol{c})$ & $-i c \sin \xi$ \\
\hline$-\triangle t^{2}\left(\boldsymbol{a} \cdot \nabla N_{i}, \boldsymbol{a} \cdot \nabla N_{j}\right) / h^{2}$ & $c^{2} \delta^{2}$ & $\mathcal{D}(\boldsymbol{\xi}, \boldsymbol{c})$ & $2 c^{2}(\cos \xi-1)$ \\
\hline
\end{tabular}

Table I. Spatial, discrete and mode transformation operators for 1D.

In Table I the discrete operators are related with the transformations for each Fourier mode and the expressions are given for linear finite elements.

For example, to determine the numerical amplification factor for the proposed time schemes and a Galerkin formulation, corresponding to $\tau=0$ in (2), the following steps are necessary:

1. From (2) find the equation that modifies each Fourier component using Table I,

$\{\mathcal{M}(\boldsymbol{\xi}) \mathbf{I}-[\mathcal{K}(\boldsymbol{\xi}, d)-\mathcal{A}(\boldsymbol{\xi}, c)-r \mathcal{M}(\boldsymbol{\xi})] \mathbf{W}\} \Delta u=[\mathcal{K}(\boldsymbol{\xi}, d)-\mathcal{A}(\boldsymbol{\xi}, c)-r \mathcal{M}(\boldsymbol{\xi})] u^{n} \mathbf{W}$.

2. Solve the linear system of equations for $\boldsymbol{z}=\left(z_{1}, z_{2}, \ldots, z_{\mathrm{n}_{\text {stages }}}\right)^{T}$ :
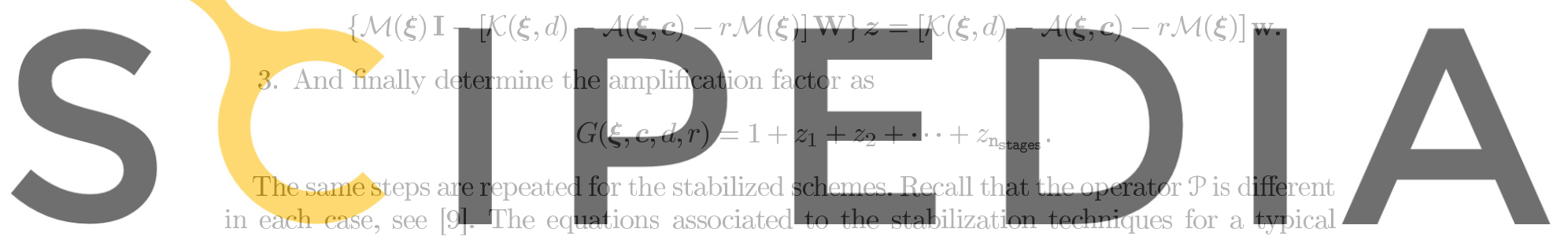

Fourier mode corresponding to a wave vector $\xi$ are as follows (note the nonlinear dependence

Register for fPed at https//www scipedia. com to download the version without the watermark

$$
\begin{gathered}
\left\{\mathcal{M}(\boldsymbol{\xi}) \overline{\mathbf{I}}-[\mathcal{K}(\boldsymbol{\xi}, d)-\mathcal{A}(\boldsymbol{\xi}, \boldsymbol{c})-r \mathcal{M}(\boldsymbol{\xi})] \overline{\mathbf{W}}-\frac{\tau}{\triangle t}\left[(\mathcal{D}(\boldsymbol{\xi}, \boldsymbol{c})+r \mathcal{A}(\boldsymbol{\xi}, \boldsymbol{c})) \overline{\mathbf{W}} \overline{\mathbf{W}}^{T} \overline{\mathbf{W}}+\mathcal{A}(\boldsymbol{\xi}, \boldsymbol{c}) \overline{\mathbf{W}}^{T}\right]\right\} \triangle \boldsymbol{u} \\
=\left\{[\mathcal{K}(\boldsymbol{\xi}, d)-\mathcal{A}(\boldsymbol{\xi}, \boldsymbol{c})-r \mathcal{M}(\boldsymbol{\xi})]+\frac{\tau}{\triangle t}\left[(\mathcal{D}(\boldsymbol{\xi}, \boldsymbol{c})+r \mathcal{A}(\boldsymbol{\xi}, \boldsymbol{c})) \mathbf{W}^{T}\right]\right\} u^{n} \mathbf{w} .
\end{gathered}
$$

Galerkin Least-Squares: $\mathcal{P}(\boldsymbol{v})=\frac{v}{\triangle t}+\mathrm{W} \mathcal{L}(\boldsymbol{v})$,

$$
\begin{aligned}
& \{\mathcal{M}(\boldsymbol{\xi}) \mathbf{I}-[\mathcal{K}(\boldsymbol{\xi}, d)-\mathcal{A}(\boldsymbol{\xi}, \boldsymbol{c})-r \mathcal{M}(\boldsymbol{\xi})] \mathbf{W} \\
- & \left.\frac{\tau}{\triangle t}\left[-\mathcal{M}(\boldsymbol{\xi}) \mathbf{I}+\mathcal{A}(\boldsymbol{\xi}, \boldsymbol{c})\left(\mathbf{W}^{T}-\mathbf{W}\right)-r \mathcal{M}(\boldsymbol{\xi})\left(\mathbf{W}^{T}+\mathbf{W}\right)+\left(\mathcal{D}(\boldsymbol{\xi}, \boldsymbol{c})-r^{2} \mathcal{M}(\boldsymbol{\xi})\right) \mathbf{W}^{T} \mathbf{W}\right]\right\} \triangle \boldsymbol{u} \\
= & \left\{[\mathcal{K}(\boldsymbol{\xi}, d)-\mathcal{A}(\boldsymbol{\xi}, \boldsymbol{c})-r \mathcal{M}(\boldsymbol{\xi})]-\frac{\tau}{\triangle t}\left[\mathcal{A}(\boldsymbol{\xi}, \boldsymbol{c})+r \mathcal{M}(\boldsymbol{\xi})-\left(\mathcal{D}(\boldsymbol{\xi}, \boldsymbol{c})-r^{2} \mathcal{M}(\boldsymbol{\xi})\right) \mathbf{W}^{T}\right]\right\} u^{n} \mathbf{w} .
\end{aligned}
$$



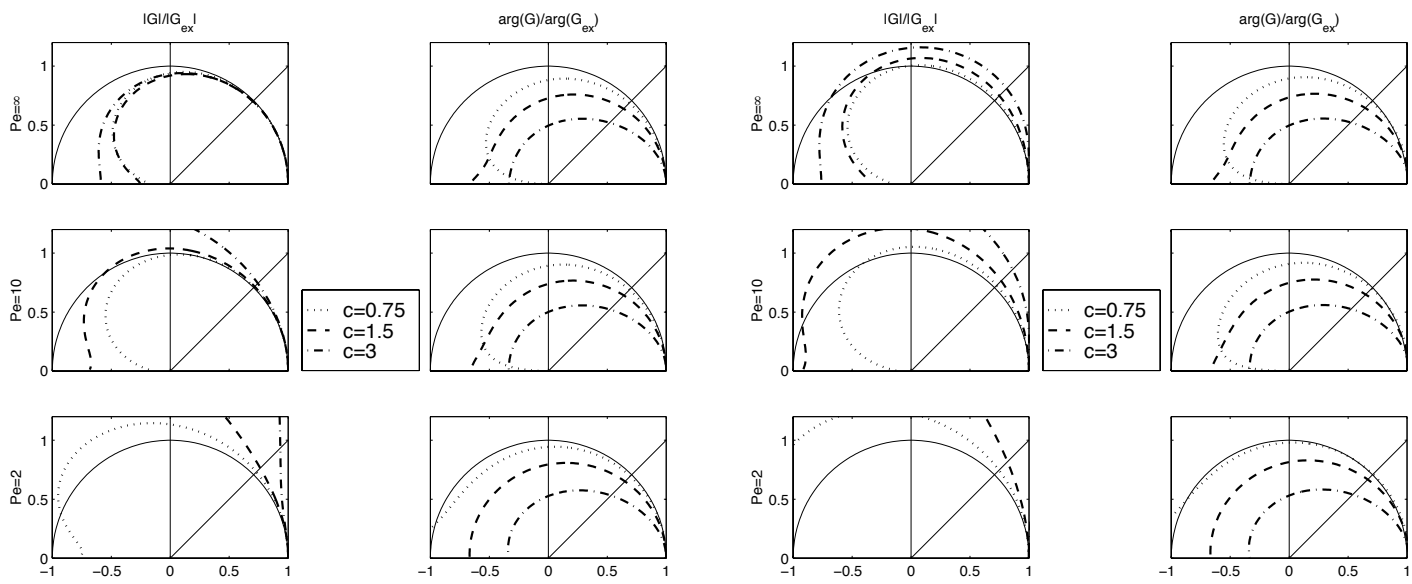

Figure 1. Accuracy properties of $R_{11}$ (Crank-Nicolson) with GLS stabilization: relative errors in amplitude and phase for $r=0$ (left) and $r=0.25$ (right) and different values of the Péclet number.
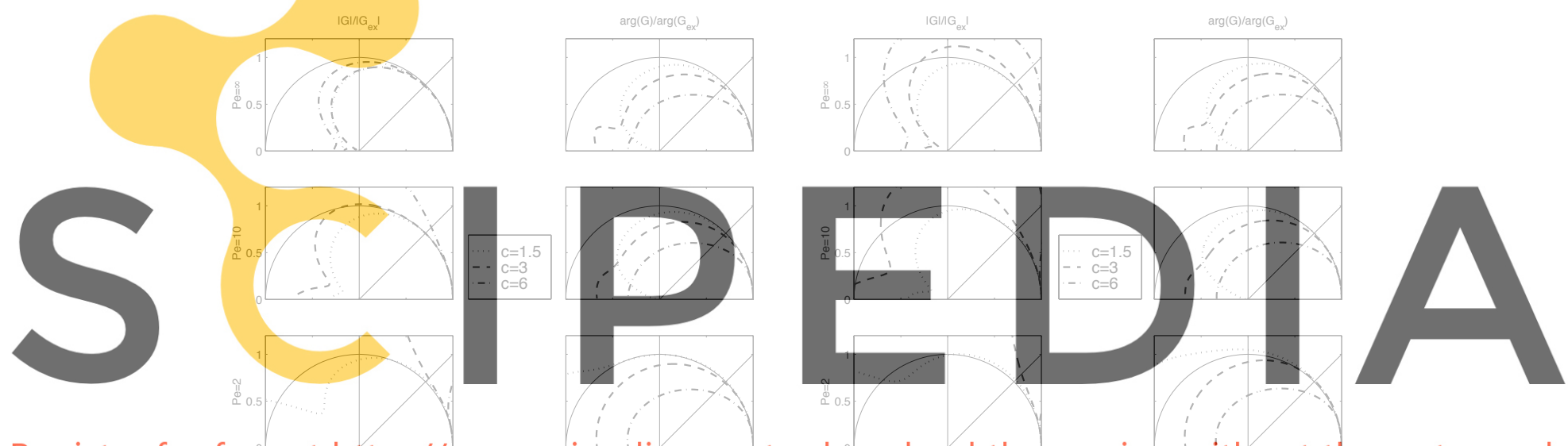

Register for free at https//www.seipedia.com to downtoad the version without the watermark

Figure 2. Accuracy properties of $R_{22}$ with GLS stabilization: relative errors in amplitude and phase for $r=0$ (left) and $r=0.5$ (right) and different values of the Péclet number.

Least-squares: $\tau \mathcal{P}(\boldsymbol{v})=\triangle t \mathbf{W} \mathcal{L}(\boldsymbol{v})$,

$$
\begin{aligned}
\{\mathcal{M}(\boldsymbol{\xi}) \mathbf{I}-[\mathcal{K}(\boldsymbol{\xi}, d)-\mathcal{A}(\boldsymbol{\xi}, \boldsymbol{c})-r \mathcal{M}(\boldsymbol{\xi})] \mathbf{W} \\
\left.-\left[(\mathcal{A}(\boldsymbol{\xi}, \boldsymbol{c})-r \mathcal{M}(\boldsymbol{\xi})) \mathbf{W}^{T}+\left(\mathcal{D}(\boldsymbol{\xi}, \boldsymbol{c})-r^{2} \mathcal{M}(\boldsymbol{\xi})\right) \mathbf{W}^{T} \mathbf{W}\right]\right\} \triangle \boldsymbol{u} \\
\quad=\left\{[\mathcal{K}(\boldsymbol{\xi}, d)-\mathcal{A}(\boldsymbol{\xi}, \boldsymbol{c})-r \mathcal{M}(\boldsymbol{\xi})]+\left[\left(\mathcal{D}(\boldsymbol{\xi}, \boldsymbol{c})-r^{2} \mathcal{M}(\boldsymbol{\xi})\right) \mathbf{W}^{T}\right]\right\} u^{n} \mathbf{w} .
\end{aligned}
$$

The fourth order (in space and for 1D) accurate formula for the stabilization parameter proposed in [15] is used $\tau=\left[\left(\frac{2 a}{h}\right)^{2}+9\left(\frac{4 \nu}{h^{2}}\right)^{2}+\sigma^{2}\right]^{-1 / 2}$. Figures 1 and 2 show the relative errors for the numerical damping, $|G(\boldsymbol{\xi}, \boldsymbol{c}, d, r)| /\left|G_{\mathrm{ex}}(\boldsymbol{\xi}, \boldsymbol{c}, d, r)\right|$, and the phase error, 
$\arg (G(\boldsymbol{\xi}, \boldsymbol{c}, d, r)) / \arg \left(G_{\mathrm{ex}}(\boldsymbol{\xi}, \boldsymbol{c}, d, r)\right)$ for different values of the Péclet number, Pe $=c /(2 d)$. All the curves are plotted in polar coordinates, the angle is $\xi$ and the radius is the relative error. From a precision point of view only the range $0 \leq \xi \leq \pi / 4$ is of interest. Thus, the limit of this sector is also depicted. Note that for Crank-Nicolson, Figure 1, the Courant numbers employed are $0.75,1.5$ and 3 . For $R_{22}$ large values of the Courant number $(1.5,3$ and 6$)$ are used to highlight its range of precision which must compensate its extra cost (double number of unknowns per node).

Only the GLS stabilization results are shown, the other stabilization techniques present little differences. The least-squares (LS) formulation introduces, as expected, more numerical diffusion for $R_{22}$ but it is almost negligible for Courant numbers up to 3. Moreover, it is only noticeable in pure convection $(\mathrm{Pe}=0)$ and this effect is beneficial in the presence of reaction. The phase accuracy is also affected by the LS stabilization but this only occurs for low Péclet numbers.

One can observe that, as expected, the stabilized methods introduce numerical damping for high frequencies ( $\xi$ close to $\pi$ ), which cannot be properly represented on the discrete mesh. Recall that Galerkin methods for pure convection do not introduce any numerical damping when combined with diagonal Padé approximations. Moreover, from a precision point of view (i.e. for dimensionless wave numbers such that $0 \leq \xi \leq \pi / 4$ ) the response (for different Péclet, Courant and reaction values) is quantitatively the same as in the Galerkin formulation. Thus, the stabilization introduced as proposed here puts the numerical diffusion where it is needed
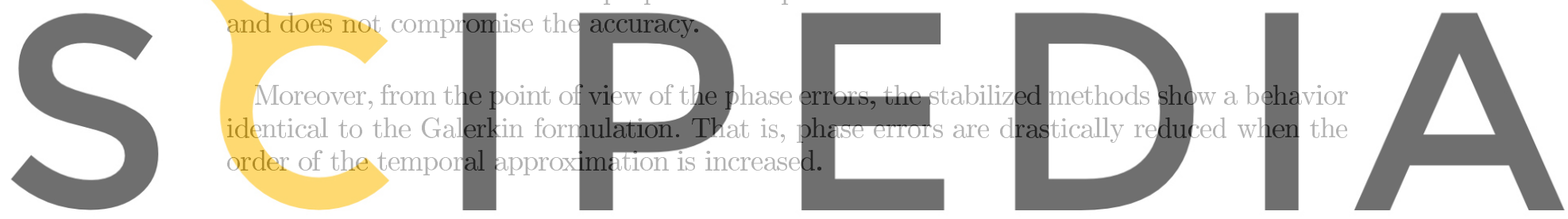

The reaction only influences the precision of the numerical damping, only for very large

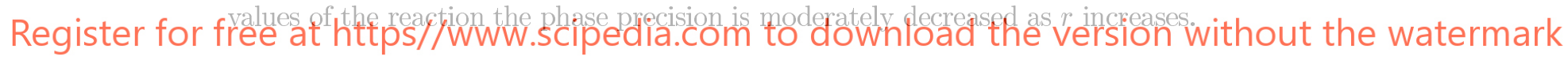

Results are only shown for Crank-Nicolson and $R_{22}$. Similar conclusions can be drawn for the sixth-order method $R_{33}$, see [8]. That is, for SUPG, GLS and SGS numerical damping is oniy introduced for high frequencies and the phase accuracy is improved for Courant numbers up to 6 . The LS stabilization, however, is over diffusive and phase errors become more noticeable. Moreover, such a formulation requires three times more unknowns than Crank-Nicolson.

An important point to be made in concluding the accuracy analysis of the proposed schemes is that the use of non-overlapping increments in the multi-stage time scheme preserves unconditional stability when the method is combined with stabilized spatial formulations. By contrast, Figure 3 shows that the classical fourth-order accurate Lobatto IIIA implicit Runge-Kutta method combined with a LS stabilization looses stability for moderate to low Péclet numbers. It also shows that the sixth-order accurate Lobatto IIIA implicit Runge-Kutta method looses stability in pure convection when combined with a GLS stabilization. This is not the case for the corresponding multi-stage schemes derived from Padé approximations. 

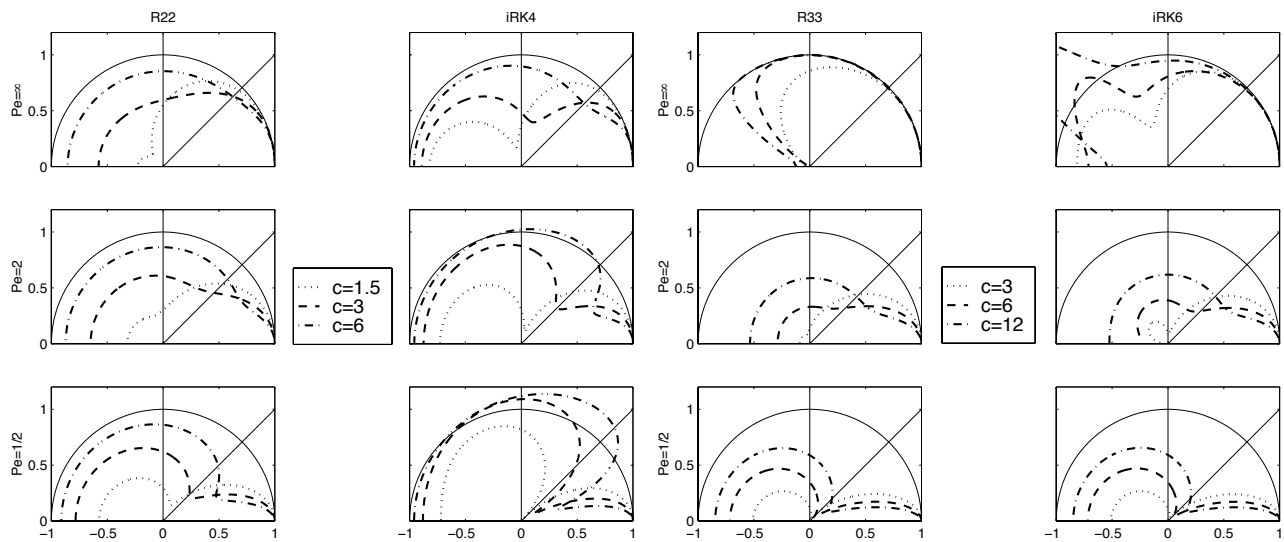

Figure 3. Modulus of the amplification factor for stabilized Pade $\left(R_{n n}\right)$ and implicit Runge-Kutta Lobatto IIIA (iRKn) methods at different values of the Péclet number: 4th order method with LS (left) and 6th order method with GLS (right).

\section{NUMERICAL EXAMPLES}

\subsection{Nonlinear propagation of a step}

In order to compare the response of $R_{11}$ (Crank-Nicolson) and $R_{22}$ with stabilized formulations,
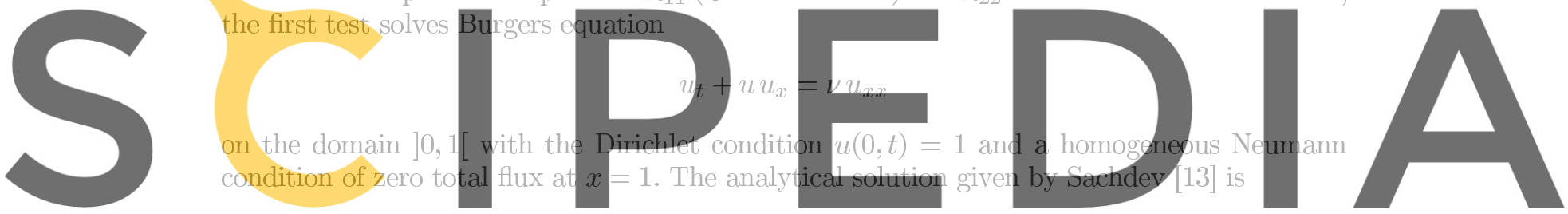

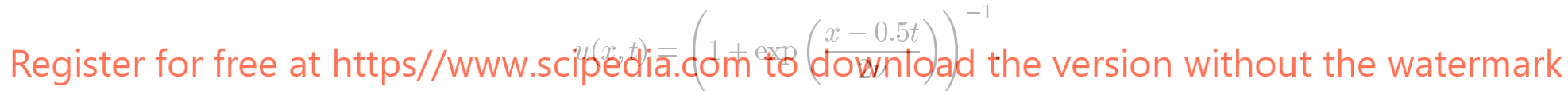

The initial condition is imposed at $t=0.02$. and a (maximum) Péclet number of 1000 is considered. A non-uniform mesh of 1000 linear elements is used. The mesh size is $h=0.002$ on $[0,0.2] \cup[0.4,1]$ and $h / 10$ on $(0.2,0.4)$. Figure 4 , where the logarithm of the absolute error is depicted at time $t=1$, shows the influence of the non-uniform mesh. The response obtained with Crank-Nicolson $(c=0.75)$ is clearly worse than $R_{22}(c=3)$ for both formulations: GLS and LS. Since the spatial mesh size is very small, this is clearly due to the inferior phase accuracy of the second-order Crank-Nicolson method. Note that $R_{22}$ runs with a time step four times larger than $R_{11}$.

\subsection{Rotating pulse}

This example, see [10], illustrates the applicability of the previously analyzed schemes to problems with internal layers and with a steady state solution. Equation (1) is solved in $\Omega=(-1,1) \times(-1,1)$ with homogeneous Dirichlet boundary conditions, and the following 

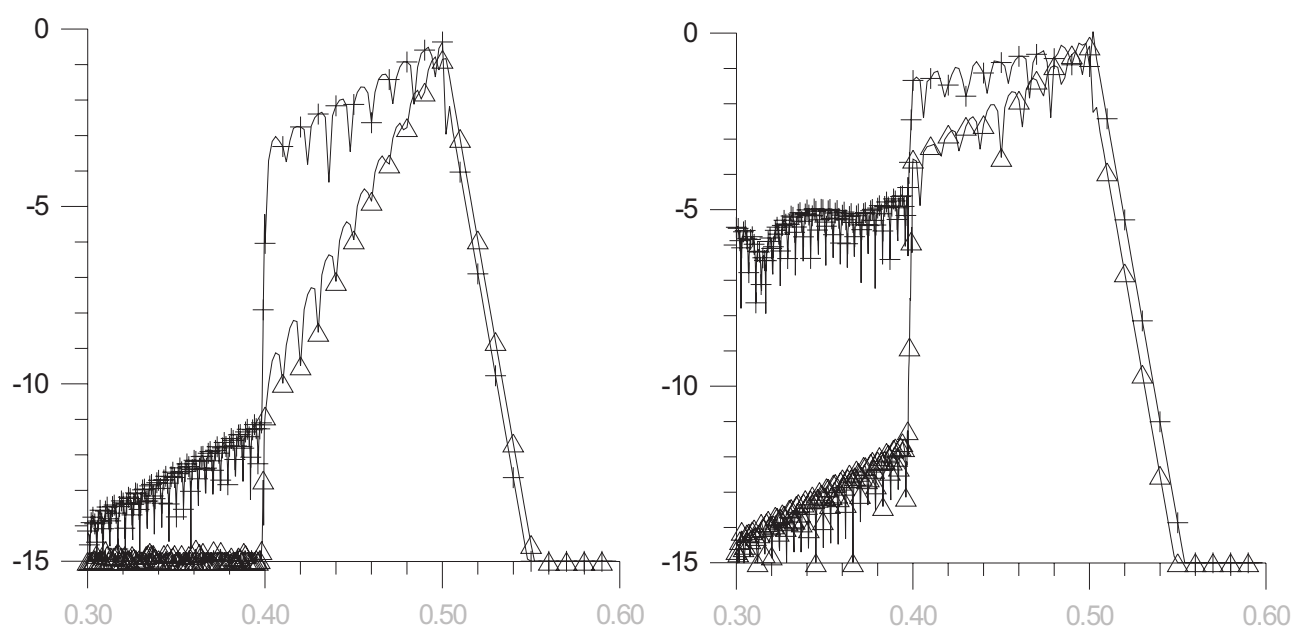

Figure 4. Logarithm of the absolute error at time $t=1$ for a LS (left) and GLS (right) stabilization. $R_{11}$ is used with $c=0.75(+)$ and $R_{22}$ with $c=3.0(\triangle)$.
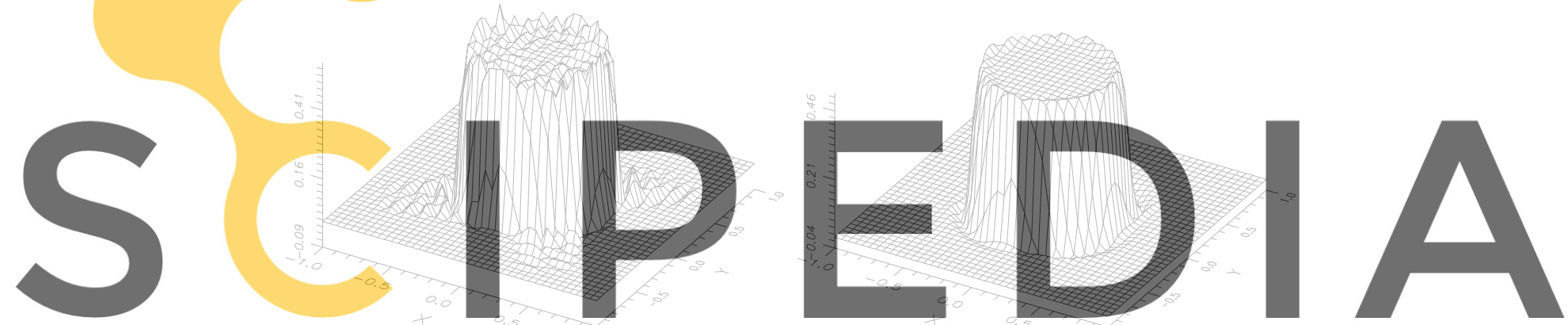

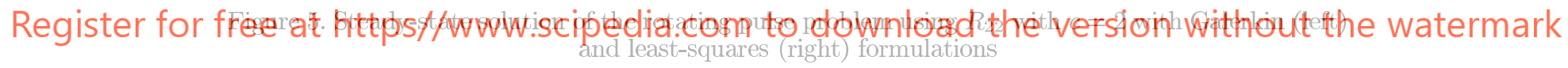

definitions: $\sigma=2, \varrho=\sqrt{x^{2}+y^{2}}$,

$$
\boldsymbol{a}=\phi(\varrho)\left(\begin{array}{c}
-y \\
x
\end{array}\right), \phi(\varrho)=\left\{\begin{array}{ll}
1-\varrho^{2} & \text { if } \varrho \leq 1, \\
0 & \text { otherwise }
\end{array} \text { and } s= \begin{cases}1 & \text { if } \varrho \leq 1 / 2 \\
0 & \text { otherwise }\end{cases}\right.
$$

The asymptotic steady state solution presents a clear pattern with $u \approx 1 / 2$ if $\varrho \leq 1 / 2$ and $u \approx 0$ otherwise, the internal layer is $\mathcal{O}(\sqrt{\nu})$ along the circle $\varrho=1 / 2$. A uniform mesh of $40 \times 40$ bilinear elements is used, Figure 5 shows the results for a Péclet number of 50000 and a least-squares stabilization. Results for other stabilized methods or for higher-order time stepping schemes $\left(R_{33}\right)$ present negligible differences. 


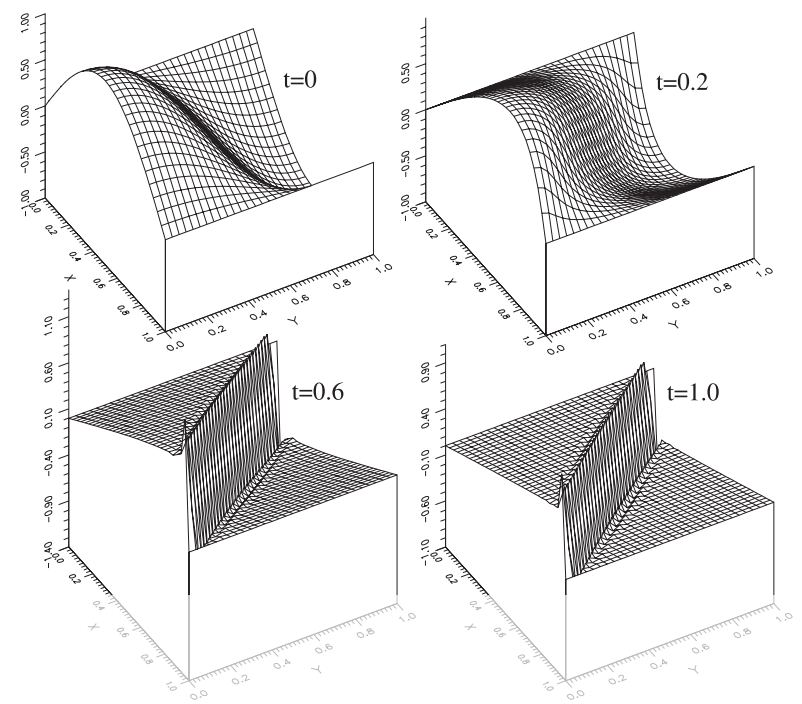

Figure 6. 2D Burgers equation: $R_{22}$ with $c=2$ and $\mathrm{Pe}=5000$ for a least-squares formulation

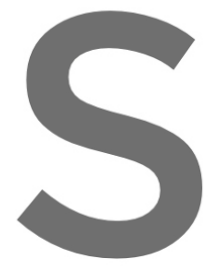

4.3. Two-dimensional Burgers equation

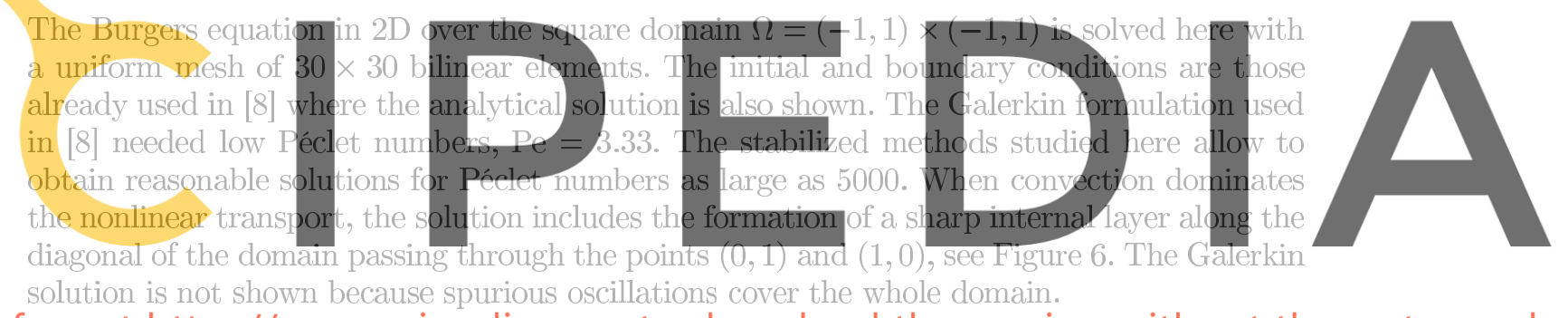
Register for free at https//www.scipedia.com to download the version without the watermark

\section{CONCLUSIONS}

Time accurate and spatially stable schemes have been proposed for the finite element solution of unsteady convection-diffusion-reaction problems. The accuracy analysis of the schemes has shown that the use of high-order implicit time stepping algorithms provides phase characteristics vastly superior to those obtained with the classical Crank-Nicolson method. On the other hand, the spatial stabilization techniques used in combination with the high-order multi-stage time marching schemes do not degrade the amplitude response in the accuracy range of the wave number. Instead, they provide damping of the high frequency modes which are badly represented on the practical finite element meshes. Moreover, the proposed time schemes based on a multi-stage factorization of Padé approximations presents superior stability properties than the corresponding Lobatto IIIA implicit Runge-Kutta methods when combined with spatial stabilization techniques. 


\section{REFERENCES}

1. W. F. Ames. Numerical methods for partial differential equations. Computer Science and Applied Mathematics. Academic Press, Inc., second edition, 1977.

2. G. Carey and B. Jiang. Least-squares finite elements for first-order hyperbolic systems. Int. J. Numer. Meth. Engng., 26:81-93, 1988.

3. R. Codina. Comparison of some finite element methods for solving the diffusion-convection-reaction equation. Comput. Methods Appl. Mech. Engrg., 156:185-210, 1998.

4. J. Donea. A Taylor-Galerkin method for convective transport problems. Int. J. Numer. Meth. Engng., 20(24):101-120, 1984.

5. J. Donea and L. Quartapelle. An introduction to finite element methods for transient advection problems. Comput. Methods Appl. Mech. Engrg., 95:169-203, 1992.

6. J. Donea, L. Quartapelle, and V. Selmin. An analysis of time discretization in the finite element solution of hyperbolic problems. J. Comput. Phys., 70:463-499, 1987.

7. J. Donea, B. Roig, and A. Huerta. High-order accurate time-stepping schemes for convection-diffusion problems. Technical Report 42, International Center for Numerical Methods in Engineering (CIMNE), Gran Capitán s/n, E-08034 Barcelona, Spain, July 1998.

8. J. Donea, B. Roig, and A. Huerta. High-order accurate time-stepping schemes for convection-diffusion problems. Comput. Methods Appl. Mech. Engrg., 182(3-4):249-275, 2000.

9. A. Huerta and J. Donea. Time-accurate solution of stabilized convection-diffusion-reaction equations: I. Time and space discretization. Comm. Numer. Methods Engrg. (submitted).

10. W.J. Layton, J.M. Maubach and P.J. Rabier. Robustness of an elementwise parallel finite element method for convection-diffusion problems. SIAM J. Sci. Comput. vol. 19. no. 6, pp. 1870-1891, 1998.

11. A. R. Mitchell and D. F. Griffiths. The Finite Difference Method in Partial Differential Equations. John Wiley \& Sons, 1980.

12. K. W. Morton. Numerical Solution of Convection-Diffusion Problems. Applied mathematics and mathematical computation, Editors R.J. Knops and K.W. Morton, volume 12. Chapman \& Hall, 1996.

13. P. L. Sachdev. Nonlinear diffusive waves. Cambridge University Press, 1987.

14. F. Shakib and T.J. R. Hughes. A new finite element formulation for computational fluid dynamics. IX. Fourier analysis of space-time Galerkin/least-squaras algorithms. Comput. Methods Appl. Mech. Engrg. $87(1): 35-58,1991$

15. F. Shakib, T. J. dynamics. X. The 89(1-3):141-219,

16. R. Wait and A. R
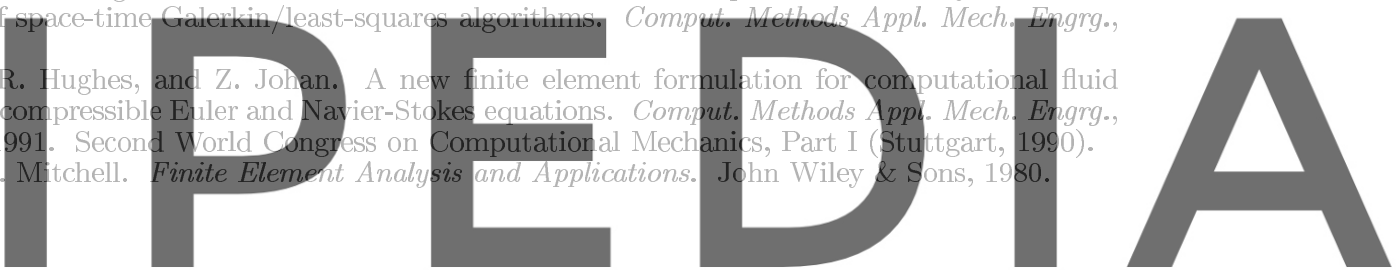